\title{
Time-Delay Robust Nonlinear Dynamic Inversion for Chaos Synchronization with Application to Secure Communications
}

\author{
Eunro Kim, ${ }^{1}$ Inseok Yang, ${ }^{2}$ and Dongik Lee $^{3}$ \\ ${ }^{1}$ Agency for Defense Development, Changwon 645-600, Republic of Korea \\ ${ }^{2}$ Institute of Biomedical Engineering Research, Kyungpook National University, Daegu 702-701, Republic of Korea \\ ${ }^{3}$ School of Electronics Engineering, Kyungpook National University, Daegu 702-701, Republic of Korea \\ Correspondence should be addressed to Dongik Lee; dilee@ee.knu.ac.kr
}

Received 1 July 2015; Accepted 8 October 2015

Academic Editor: Mohamed Djemai

Copyright (C) 2015 Eunro Kim et al. This is an open access article distributed under the Creative Commons Attribution License, which permits unrestricted use, distribution, and reproduction in any medium, provided the original work is properly cited.

\begin{abstract}
The time-delay robust nonlinear dynamic inversion (TDRNDI) control technique is proposed to synchronize time-delay Chen systems. The time-delay Chen circuit is simple but exhibits complex irregular (chaotic) behavior. For this reason, this circuit can be efficiently used to encrypt messages for secure communication. In this paper, the nonlinear control-based chaos synchronization problem is considered. The proposed TDRNDI controller is a modified version of a robust nonlinear dynamic inversion (RNDI) applicable to chaotic systems, including time-delay systems. The performance and feasibility of the proposed TDRNDI controller are demonstrated by conducting numerical simulations with application to a secure communication network.
\end{abstract}

\section{Introduction}

CHAOS is a widely observed phenomenon in nature. Because chaotic phenomena are aperiodic, unpredictable, and sensitive to initial conditions, the control and motion synchronization of chaotic systems have been regarded as challenging problems in science and engineering [1]. One excellent example of these challenging problems is secure communication. Relative to modern digital communication, which is based on linear theory, chaos communication provides a high level of security because of its aperiodicity and unpredictability [26]. That is, once a message is encrypted by chaotic circuits, the message cannot be broken or decrypted due to the difficulties associated with predicting the pattern of the encrypted message. This paper examines the chaos synchronization problem in the context of secure communication. Chaos synchronization refers to a motion control technique that allows two or more chaotic systems to exhibit identical behaviors [1-15]. Figure 1 depicts the behaviors of two chaotic systems with different initial conditions: (a) without synchronization and (b) with synchronization [15]. As indicated in Figure 1, the use of a controller can allow two chaotic systems with different irregular motions to display the same behavior. In a secure communication system based on a chaotic circuit, chaos synchronization is used by a receiver to decrypt a message. Various methods based on nonlinear control theory have been proposed for synchronizing of chaotic systems; these methods include active control [7,8], passive control $[3,9,10]$, backstepping control $[4,11,12]$, sliding mode control (SMC) $[5,6,13,14]$, and robust nonlinear dynamic inversion (RNDI) [15].

In [15-17], Yang et al. proposed the RNDI controller. By adopting a switching input into conventional nonlinear dynamic inversion (NDI), the RNDI controller improves upon the poor robustness of NDI. The switching input utilized for RNDI is designed in accordance with the design concept of SMC, which is regarded as a nonlinear robust controller. In SMC, a switching input is used to generate boundaries that ensure robustness. Based on the similar characteristics of NDI and SMC, robustness can be obtained by adopting this switching input into NDI [16]. Thus, the RNDI controller can exhibit both robustness and the advantages of NDI, including not only the elimination of a need for gain-scheduled controllers but also ease of design and implementation according to the similar concept to classical control method. Due to these advantages, it is reasonable that the RNDI is considered as the primary controller for the chaos synchronization problem. However, the RNDI 

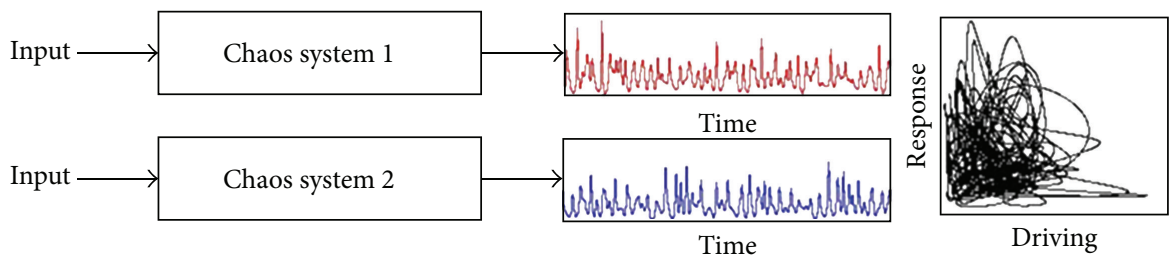

(a) Without synchronization

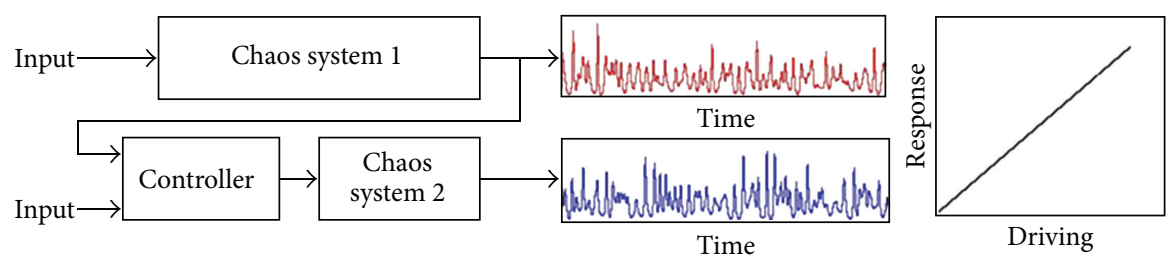

(b) With synchronization

FIGURE 1: Behaviors of two chaotic systems [15].

proposed in $[16,17]$ cannot be directly applied to time-delay chaotic systems such as the time-delay Chen circuit. In this paper, a time-delay RNDI (TDRNDI) controller is proposed for the synchronization of time-delay chaotic systems.

This paper is organized as follows. The time-delay Chen system and synchronization problem are introduced in Section 2. In Section 3, the TDRNDI controller is proposed and the stability of the proposed controller is analyzed. In Section 4, the performance of the proposed controller is evaluated by conducting numerical simulations with application to a secure communication system designed using the timedelay Chen circuit. Finally, concluding remarks are provided in Section 5.

\section{The Time-Delay Chen System}

In this paper, the time-delay Chen chaotic system is considered for secure communication. This system can be designed by adding a time-delay feedback term to the original Chen chaotic system [18]. In [18], it was reported that the original Chen system, if designed with certain parameters, exhibits nonchaotic motion. By adding the time-delay feedback term to the original Chen system, Ren and $\mathrm{Li}$ proposed the following new chaotic system, which is known as the timedelay Chen system [18]:

$$
\begin{aligned}
& \dot{x}_{1}(t)=a\left[x_{2}(t)-x_{1}(t)\right], \\
& \dot{x}_{2}(t)=(c-a) x_{1}(t)-x_{1}(t) x_{3}(t)+c x_{2}(t), \\
& \dot{x}_{3}(t)=x_{1}(t) x_{2}(t)-b x_{3}(t)+k\left[x_{3}(t)-x_{3}(t-\tau)\right],
\end{aligned}
$$

where $\tau$ is a time-delay constant and $k$ is a time-delay feedback gain. The chaotic attractor and state trajectories of (1) with parameters $a=35, b=3$, and $c=18.5$ are presented in Figures 2 and 3, respectively.

Generally, chaos synchronization is to apply external forces that cause states of two or more chaotic systems with different initial conditions to exhibit the same motions. In secure communication, the chaos synchronization technique is used by a receiver to decrypt a secured message. Figure 4 illustrates secure communication using the time-delay Chen circuits. In this figure, if the sender encrypts the message, then the encrypted message can only be decrypted by the receiver, which has synchronization capability, due to the sensitivity of chaotic circuit to initial conditions. A simple example of message encryption using the time-delay Chen chaotic circuit with the initial conditions $x_{1}(0)=1 \mathrm{~V}$ (Volt), $x_{2}(0)=0 \mathrm{~V}$, and $x_{3}(0)=1 \mathrm{~V}$ is depicted in Figure 5: (a) the original message $m_{o}$ is designed by utilizing a binary pulse with a period of $2 \mathrm{sec}$, and (b) the message encryption result is obtained using a simple encrypting function $\varphi_{e}: \mathbf{R}^{3} \times[0,1] \rightarrow \mathbf{R}$; for example,

$$
\varphi_{e}\left(\mathbf{R}^{3}, m_{o}\right)=x_{1}+x_{2}+x_{3}+m_{o}
$$

Once the encrypted message is transmitted through a public channel, the receiver can decrypt it by employing the decryption function $\varphi_{d}: \mathbf{R}^{3} \times \mathbf{R} \rightarrow \mathbf{R}$; for example, $\varphi_{d}\left(\mathbf{R}^{3}, m_{e}\right)=m_{d}=m_{e}-y_{1}-y_{2}-y_{3}$. In actual applications, because a great deal of noise is inserted during the course of encrypting, transmitting, sensing, and decrypting messages, the image of the decryption function must be a real number rather than a binary pulse. To obtain the original message, the receiver must have real-time information regarding the states $\left[x_{1}, x_{2}, x_{3}\right]^{T}$. However, the acquisition of real-time state data from the sender is impossible due to security considerations; instead, only limited data can be obtained by the receiver. Thus, the receiver must estimate all states from limited information. A nonlinear control method can be efficiently applied to estimate the unknown states, that is, to achieve chaos synchronization. Generally, the basis chaotic system used to encrypt the original message like (1) is called the drive system whereas the controlled system is defined as the response system. In this paper, the following system is regarded as the response system:

$$
\begin{aligned}
& \dot{y}_{1}(t)=a\left[y_{2}(t)-y_{1}(t)\right], \\
& \dot{y}_{2}(t)=(c-a) y_{1}(t)-y_{1}(t) y_{3}(t)+c y_{2}(t)+u_{2}(t),
\end{aligned}
$$




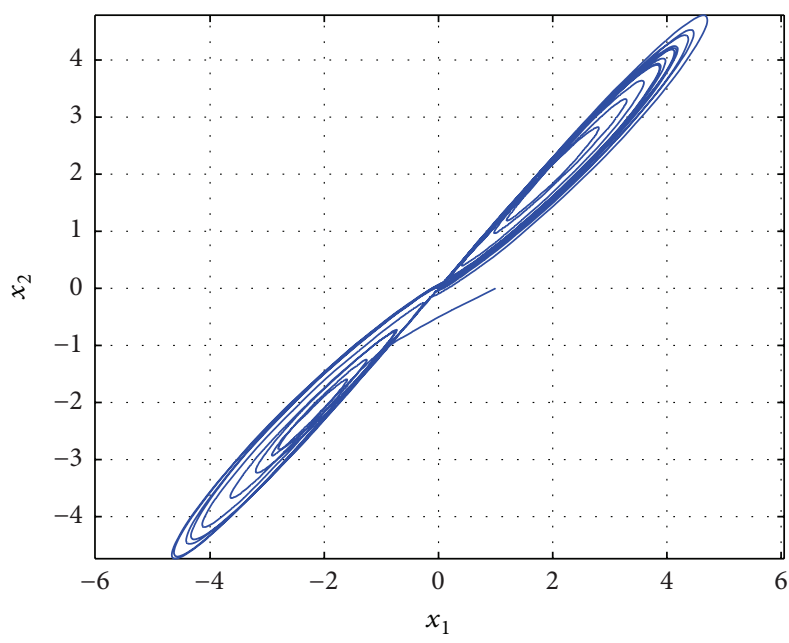

(a) $x_{1}-x_{2}$

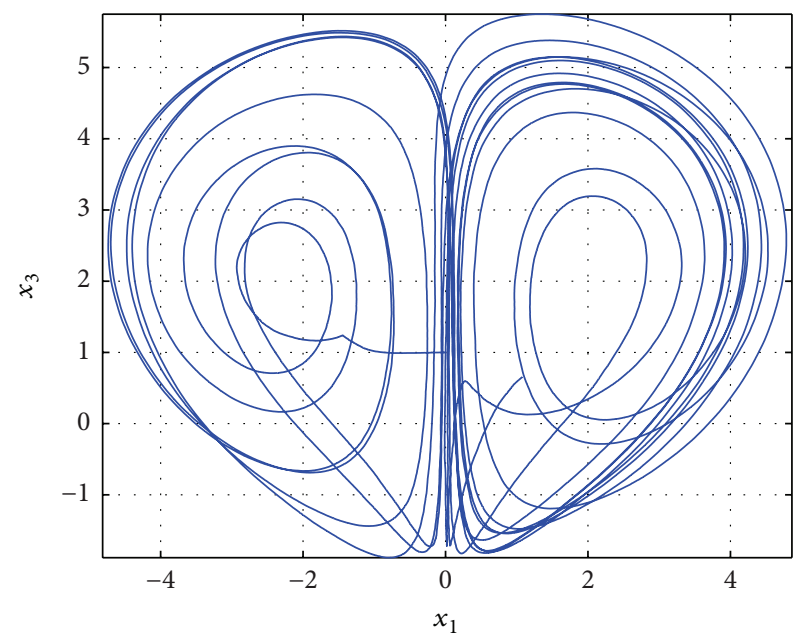

(b) $x_{1}-x_{3}$

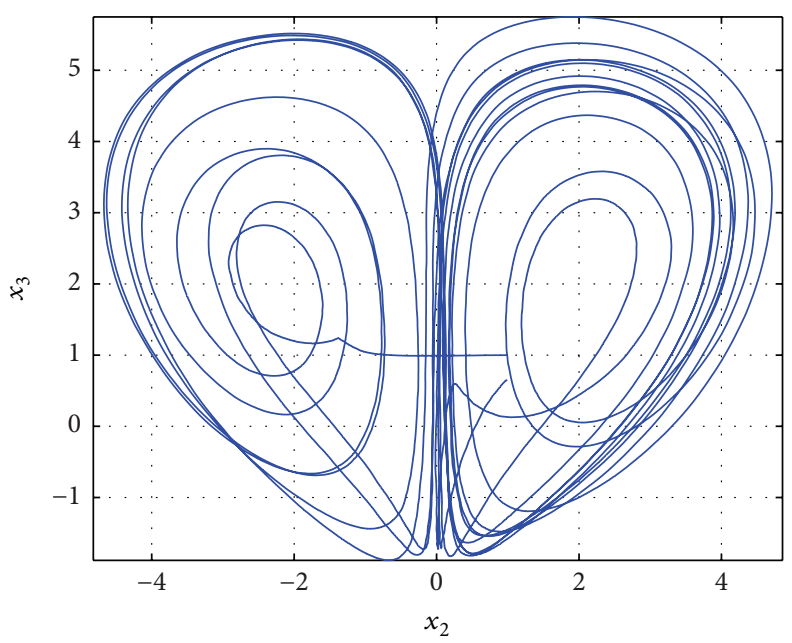

(c) $x_{2}-x_{3}$

FIgURE 2: The time-delay Chen chaotic attracter.

$$
\begin{aligned}
\dot{y}_{3}(t)= & y_{1}(t) y_{2}(t)-b y_{3}(t)+k\left[y_{3}(t)-y_{3}(t-\tau)\right] \\
& +u_{3}(t)
\end{aligned}
$$

where $u_{2}(t)$ and $u_{3}(t)$ are inputs for controlling the states $y_{2}(t)$ and $y_{3}(t)$, respectively.

By designing the controllers $u_{2}(t)$ and $u_{3}(t)$, the receiver can estimate the states $\left[x_{1}(t), x_{2}(t), x_{3}(t)\right]^{T}$ and, finally, recover the original message.

\section{The Proposed Time-Delay Robust Nonlinear Dynamic Inversion}

In this section, the TDRNDI for controlling chaotic systems is proposed. To improve on the poor robustness of NDI, Yang et al. proposed the RNDI, which involved the addition of a switching input to the conventional NDI [16, 17]. This switching term generates boundaries within which the controlled states can reach the references. Due to its simple design, robustness, and adaptation to nonlinear control systems, the RNDI can serve as the primary controller for chaos synchronization. In this paper, the RNDI controller is modified to achieve chaos synchronization for time-delay chaotic systems.

Figure 6 presents a block diagram of the TDRNDI controller. As indicated in this figure, the proposed TDRNDI consists of two blocks: desired dynamics and dynamic inversion. In the desired dynamics block, control variables are selected, and the desired dynamics are calculated. In this paper, $y_{2}$ and $y_{3}$ in (3) are selected as control variables, and the referenced desired dynamics are designed as follows (Figure 6(b)):

$$
\begin{aligned}
& \dot{y}_{2, \text { des }}(t)=\sigma_{2}\left[x_{2}(t)-y_{2}(t)\right], \\
& \dot{y}_{3, \text { des }}(t)=\sigma_{3}\left[x_{3}(t)-y_{3}(t)\right],
\end{aligned}
$$

where $\sigma_{2} \in \mathbf{R}$ and $\sigma_{3} \in \mathbf{R}$ are the gains of the desired dynamics. In fact, various types of desired dynamics proposed in the literature [19] can be efficiently used to calculate these 


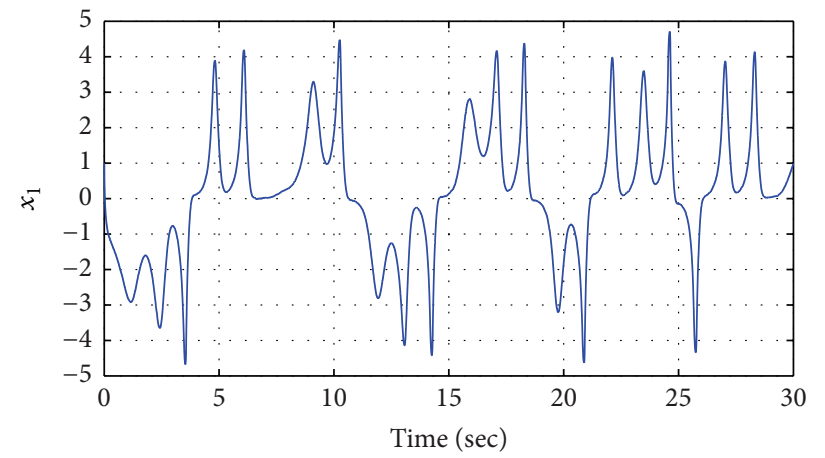

(a)

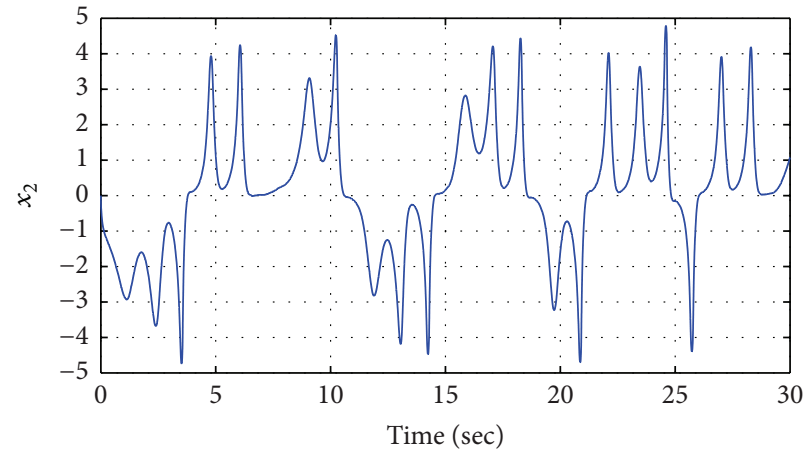

(b)

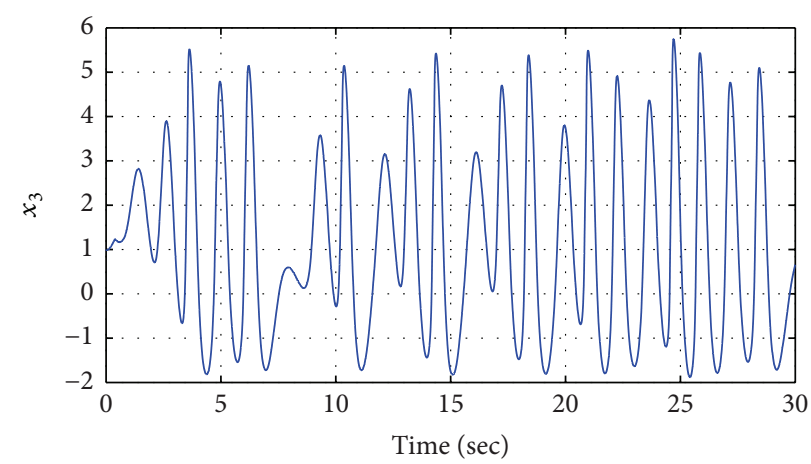

(c)

FIGURE 3: The state trajectories of the time-delay Chen system.

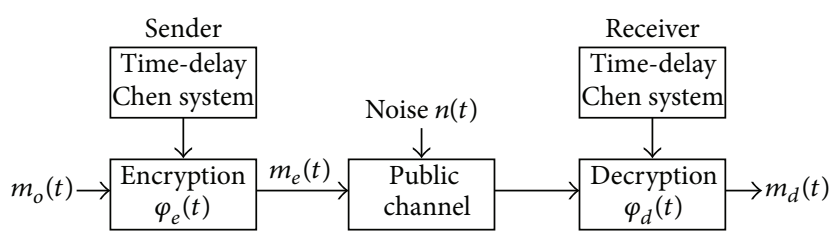

FIGURE 4: Secure communication using the time-delay Chen system.

desired dynamics. Once the desired dynamics are analyzed, the original system dynamics are removed in the dynamic inversion block; that is, by inverting the plant dynamics, the inner closed-loop transfer function is replaced into an integrator (Figure 6(a)). Consequently, the original dynamics are replaced with the user-designed desired dynamics.

Combining the above two blocks in Figure 6(a), the TDRNDI is proposed as follows:

$$
\begin{aligned}
& u_{2}(t) \\
& =\dot{y}_{2, \mathrm{des}}(t)-\left[(c-a) y_{1}(t)-y_{1}(t) y_{3}(t)+c y_{2}(t)\right] \\
& +k_{2} \operatorname{sign}\left(y_{2, \mathrm{des}}(t)-y_{2}(t)\right), \\
& u_{3}(t) \\
& =\dot{y}_{3, \mathrm{des}}(t) \\
& -\left[y_{1}(t) y_{2}(t)-b y_{3}(t)+k\left[y_{3}(t)-y_{3}(t-\tau)\right]\right] \\
& +k_{3} \operatorname{sign}\left(y_{3, \mathrm{des}}(t)-y_{3}(t)\right) \text {, }
\end{aligned}
$$

where $k_{2}>0$ and $k_{3}>0$ are the switching gains.
Theorem 1. Consider the following disturbed response system:

$$
\begin{aligned}
\dot{y}_{2}(t)= & (c-a) y_{1}(t)-y_{1}(t) y_{3}(t)+c y_{2}(t)+u_{2}(t) \\
& +n_{2}(t), \\
\dot{y}_{3}(t)= & y_{1}(t) y_{2}(t)-b y_{3}(t)+k\left[y_{3}(t)-y_{3}(t-\tau)\right] \\
& +u_{3}(t)+n_{3}(t),
\end{aligned}
$$

where $n_{i}(t)(i=2,3)$ is disturbance. If $\left|n_{i}(t)\right|<k_{i}$ holds for each $i$, then system (6) controlled by the TDRNDI (5) is globally stable.

Proof. To verify the stability of the proposed TDRNDI controller, the Lyapunov criterion is employed. The following Lyapunov candidate is selected:

$$
V=\frac{1}{2}\left[\left(y_{2, \mathrm{des}}(t)-y_{2}(t)\right)^{2}+\left(y_{3, \mathrm{des}}(t)-y_{3}(t)\right)^{2}\right] .
$$

The derivative can then be analyzed as follows:

$$
\begin{aligned}
\dot{V} & =\left(y_{2, \mathrm{des}}(t)-y_{2}(t)\right)\left(\dot{y}_{2, \mathrm{des}}(t)-\dot{y}_{2}(t)\right)+\left(y_{3, \mathrm{des}}(t)\right. \\
& \left.-y_{3}(t)\right)\left(\dot{y}_{3, \mathrm{des}}(t)-\dot{y}_{3}(t)\right)=\left(y_{2, \mathrm{des}}(t)-y_{2}(t)\right) \\
& \cdot\left[\dot{y}_{2, \mathrm{des}}(t)-\left\{(c-a) y_{1}(t)-y_{1}(t) y_{3}(t)+c y_{2}(t)\right.\right. \\
& \left.\left.+u_{2}(t)+n_{2}(t)\right\}\right]+\left(y_{3, \mathrm{des}}(t)-y_{3}(t)\right)\left[\dot{y}_{3, \mathrm{des}}(t)\right. \\
& -\left\{y_{1}(t) y_{2}(t)-b y_{3}(t)+k\left[y_{3}(t)-y_{3}(t-\tau)\right]\right. \\
& \left.\left.+u_{3}(t)+n_{3}(t)\right\}\right] .
\end{aligned}
$$




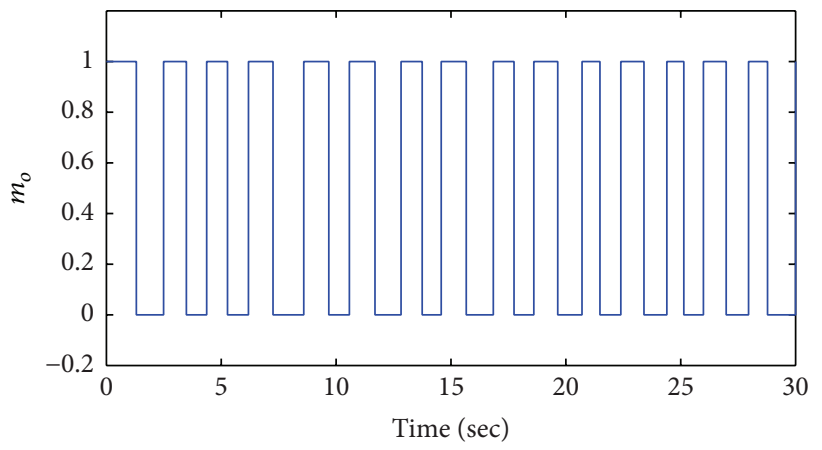

(a) Original message

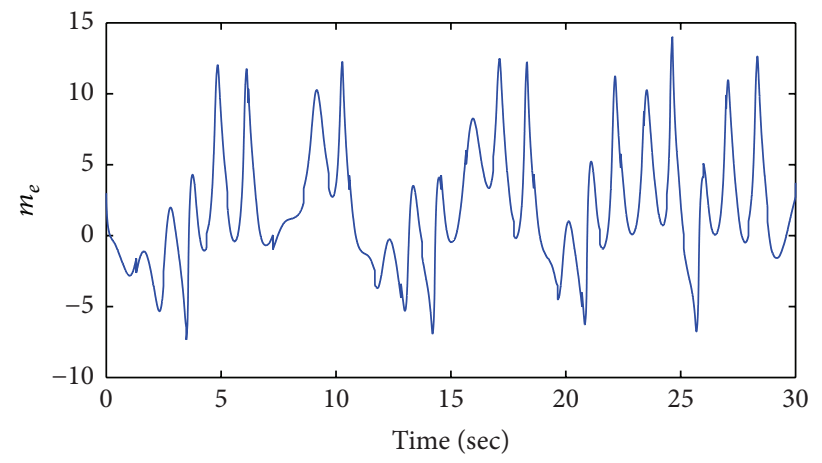

(b) Encrypted message

Figure 5: Message encryption example.

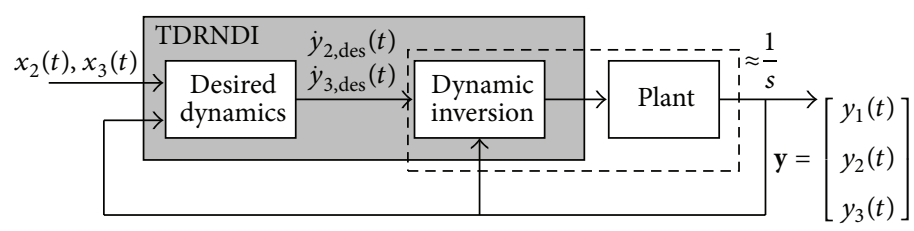

(a) Block diagram of the proposed time-delay robust nonlinear dynamic inversion

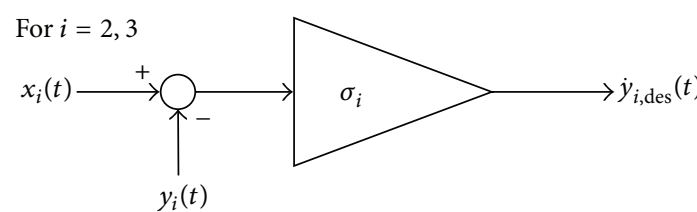

(b) Block diagram of the desired dynamics

FIGURE 6: Block diagram of the proposed TDRNDI controller.

By inserting the TDRNDI control law (5) into (8), the derivative yields

$$
\begin{aligned}
\dot{V} & =\left(y_{2, \text { des }}(t)-y_{2}(t)\right)\left[-n_{2}(t)\right. \\
& \left.-k_{2} \operatorname{sign}\left(y_{2, \text { des }}(t)-y_{2}(t)\right)\right]+\left(y_{3, \text { des }}(t)-y_{3}(t)\right) \\
& \cdot\left[-n_{3}(t)\right. \\
& \left.-k_{3} \operatorname{sign}\left(y_{3, \text { des }}(t)-y_{3}(t)\right)\right] \\
& =\left[-n_{2}(t)\left(y_{2, \text { des }}(t)-y_{2}(t)\right)\right. \\
& \left.-k_{2}\left|y_{2, \text { des }}(t)-y_{2}(t)\right|\right] \\
& +\left[-n_{3}(t)\left(y_{3, \text { des }}(t)-y_{3}(t)\right)\right. \\
& \left.-k_{3}\left|y_{3, \text { des }}(t)-y_{3}(t)\right|\right] \leq\left(\left|n_{2}(t)\right|-k_{2}\right) \mid y_{2, \text { des }}(t) \\
& -y_{2}(t)\left|+\left(\left|n_{3}(t)\right|-k_{3}\right)\right| y_{3, \text { des }}(t)-y_{3}(t) \mid .
\end{aligned}
$$

Because $\left|n_{i}(t)\right|<k_{i}$ holds for each $i$, the derivative of the Lyapunov candidate is always negative; that is, $d V / d t<0$. Thus, the trajectories of $y_{2}$ and $y_{3}$, which are controlled by the proposed TDRNDI controller, can converge to $x_{2}$ and $x_{3}$, respectively. Hence, the disturbed system controlled by (5) is globally stable.

The remaining task for achieving synchronization is to verify the convergence of $y_{1}$ to $x_{1}$. For $i=1,2$, set $e_{i}(t)=x_{i}(t)-y_{i}(t)$. From (1) and (3), the derivative of error dynamics becomes

$$
\begin{aligned}
\dot{e}_{1}(t) & =\dot{x}_{1}(t)-\dot{y}_{1}(t) \\
& =a\left[x_{2}(t)-x_{1}(t)\right]-a\left[y_{2}(t)-y_{1}(t)\right] \\
& =a\left[e_{2}(t)-e_{1}(t)\right] .
\end{aligned}
$$

Because $y_{2}(t) \rightarrow x_{2}(t)$ as $t \rightarrow \infty, \dot{e}_{1}(t)$ converges to $-a e_{1}(t)$; that is, $e_{1}(t) \rightarrow \exp (-a t)$. Moreover, since $a=35>0$ for the considered time-delay Chen system, $e_{1}(t)$ converges to zero as $t \rightarrow \infty$; consequently, $y_{1}(t)$ converges to $x_{1}(t)$.

Hence, using the proposed TDRNDI controller, the states of the response system can track the referenced motions of the drive system. As a result, the synchronization of the two chaotic systems can be achieved.

\section{Simulation Results}

In this section, the performance of the proposed TDRNDI controller is evaluated by conducting numerical simulations with application to secure communication. In this paper, the time-delay Chen chaotic system is used to encrypt/decrypt a message for secure communication. The parameters of the considered time-delay Chen system are assumed to be $a=$ $35, b=3$, and $c=18.5$. The initial conditions for the drive and response systems are assumed to be $\left[x_{1}(0), x_{2}(0), x_{3}(0)\right]^{T}$ $=[1,0,1]^{T}(\mathrm{~V})$ and $\left[y_{1}(0), y_{2}(0), y_{3}(0)\right]^{T}=[0,0,0]^{T}(\mathrm{~V})$, respectively. It is assumed that the time-delay constant $\tau$ is 0.03 and the time-delay feedback gain $k$ is 0.2 . Moreover, 


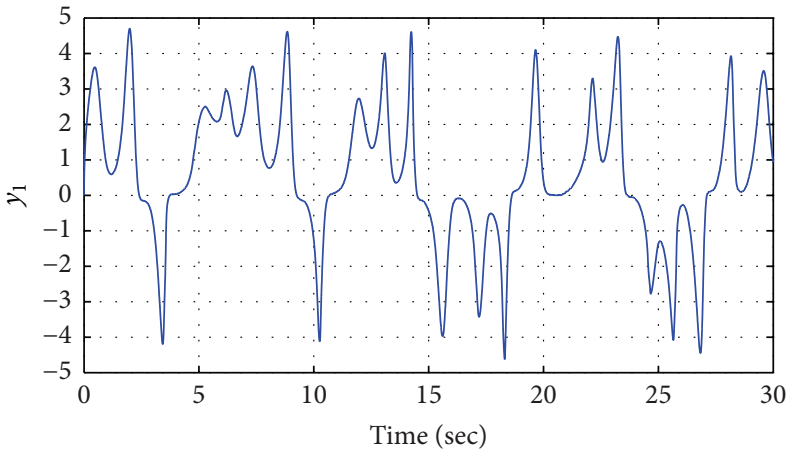

(a) Trajectory of $y_{1}$

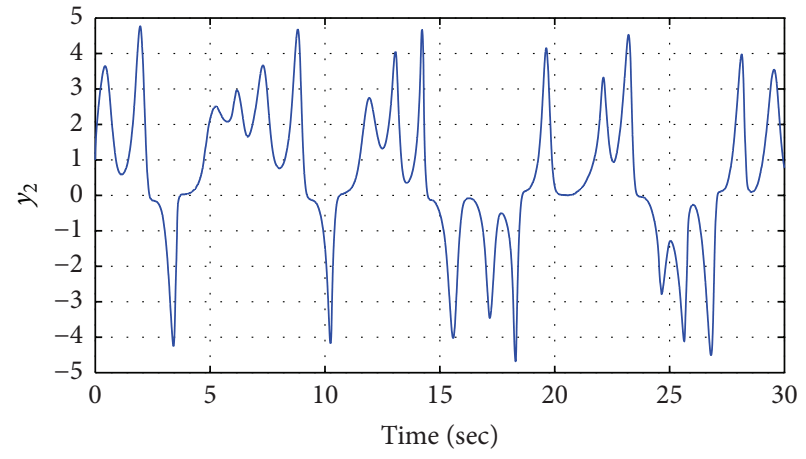

(b) Trajectory of $y_{2}$

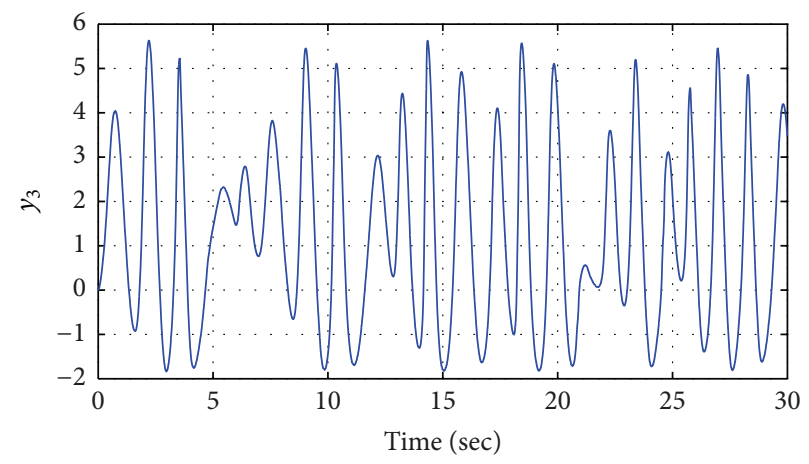

(c) Trajectory of $y_{3}$

FIGURE 7: Trajectories of the uncontrolled response system.

$5 \%$ of the random noise $n(t)$ is assumed to be injected into the communication channel. To verify the performance of the proposed controller, the results of chaos synchronization are provided first, and then message decryption results are followed.

Figures 7-10 present the results of chaos synchronization using the proposed TDRNDI controller. The trajectories of the drive system states are depicted in Figure 3. These trajectories are considered as the references that the states of the response system must track to achieve chaos synchronization. The behaviors of the response system are indicated in Figures 7 (uncontrolled) and 9 (controlled). A comparison of Figures 3 and 7 reveals that the states of the drive and response systems exhibit different motions; in other words, the uncontrolled results cannot track the referenced trajectories due to the distinct initial conditions. This result can clearly be observed in Figure 8, which describes the error dynamics between the states of the drive and uncontrolled response systems. In Figure 8, the maximum errors of $e_{1}$ and $e_{2}$ are $10 \mathrm{~V}$.

Figure 9 depicts the behaviors of the response system controlled by the proposed TDRNDI. After control laws have been applied, the response system states $y_{2}$ and $y_{3}$ are forced to track the referenced states $x_{2}$ and $x_{3}$, respectively. Moreover, the state $y_{1}$ can accurately estimate the state $x_{1}$. As a result, the response system states can track the drive system states with tracking errors of less than $0.05 \mathrm{~V}$ (Figure 10). Hence, using the proposed TDRNDI controller, chaos synchronization can be quickly and accurately attained.
As demonstrated by the results of Figures 9-10, the states of the response system can track the referenced trajectory of the drive system with high accuracy. These results indicate that the receiver can estimate the encryption states; consequently, the transmitted message can be decrypted by applying the decryption function $\varphi_{d}\left(\mathbf{R}^{3}, m_{e}\right)$. Figure 11 presents the simulation results for message decryption. In this figure, the original message can be obtained. Due to the injected channel noise, a small quantity of noise (less than $2 \%$ ) causes perturbations in the decrypted signal.

\section{Concluding Remarks}

In this paper, the TDRNDI controller has been proposed to synchronize time-delay chaotic systems. The proposed TDRNDI is a version of the conventional RNDI controller that has been modified to be suitable for time-delay systems. Therefore, the TDRNDI can exhibit the advantages of conventional RNDI, which include ease of design and implementation, with concepts similar to the principles of classical control methods; the elimination of gain-scheduling requirements; and robustness. Simulation results with application to a secure communication system demonstrate the performance and feasibility of the proposed TDRNDI controller. For future work, a secure communication system based on the proposed TDRNDI controller will be experimentally validated. 


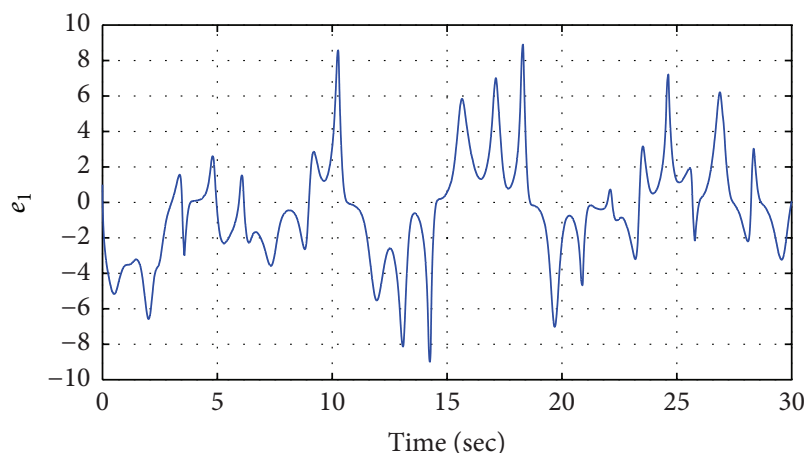

(a) Error between $x_{1}$ and $y_{1}$

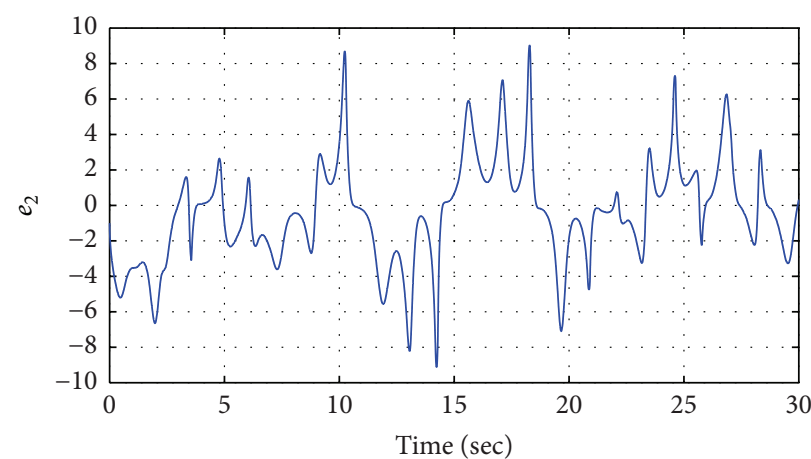

(b) Error between $x_{2}$ and $y_{2}$

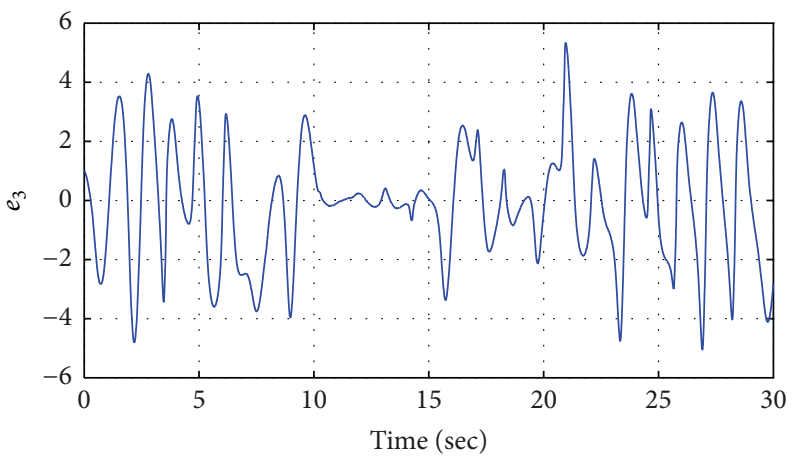

(c) Error between $x_{3}$ and $y_{3}$

FIGURE 8: Error dynamics between the drive and uncontrolled response system.

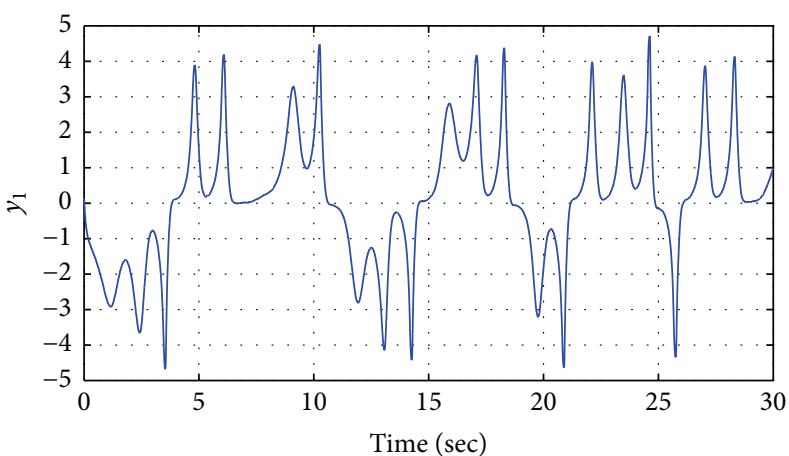

(a) Trajectory of $y_{1}$

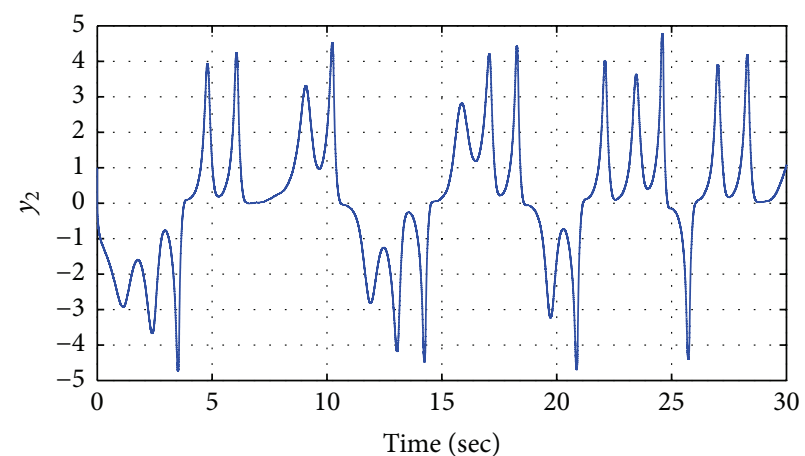

(b) Trajectory of $y_{2}$

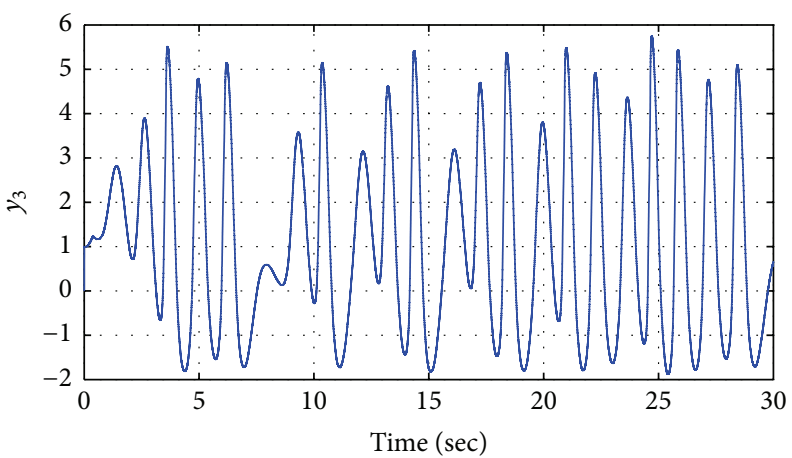

(c) Trajectory of $y_{3}$

FIgURE 9: Trajectories of the controlled response system. 


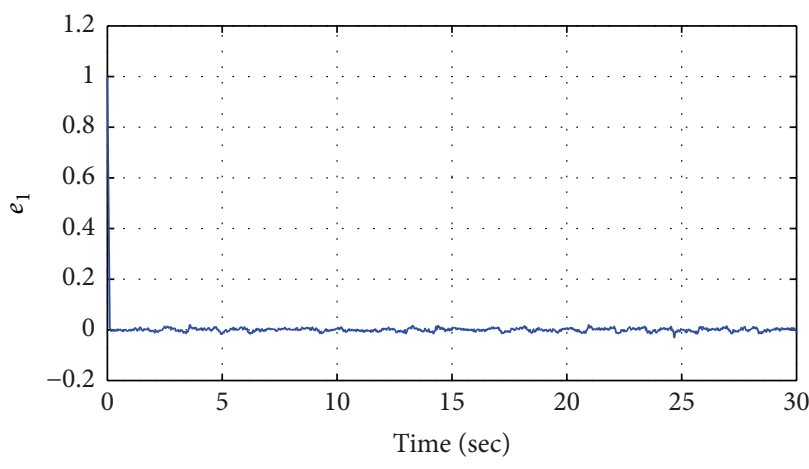

(a) Error between $x_{1}$ and $y_{1}$

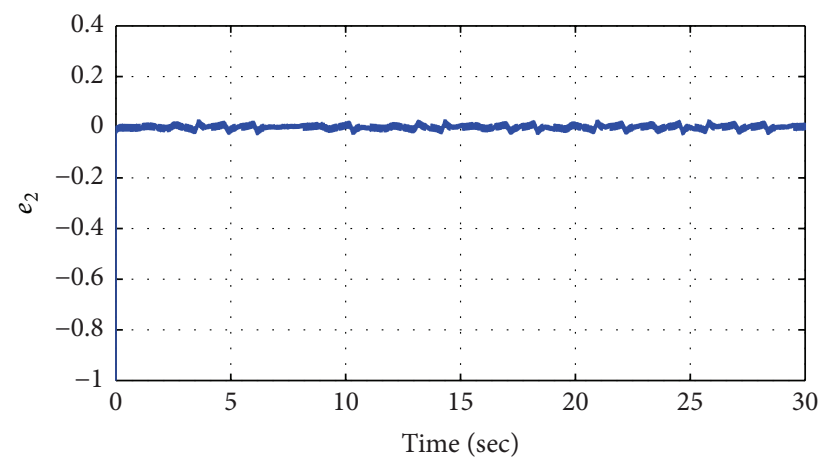

(b) Error between $x_{2}$ and $y_{2}$

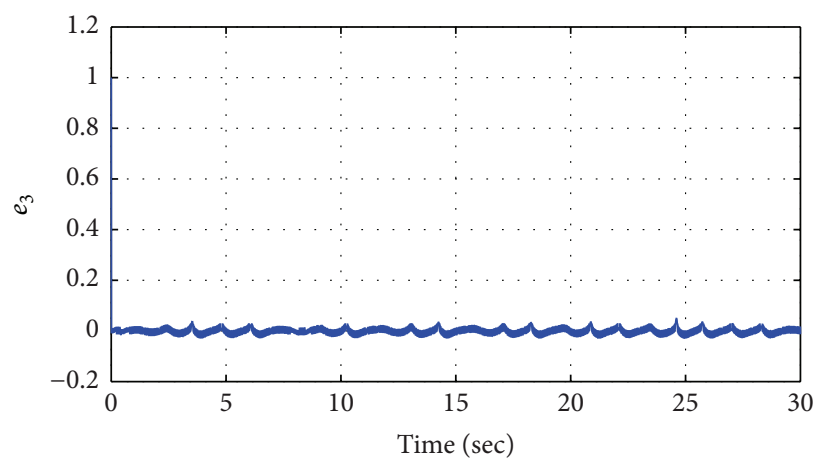

(c) Error between $x_{3}$ and $y_{3}$

FIGURE 10: Error dynamics between the drive and controlled response system.

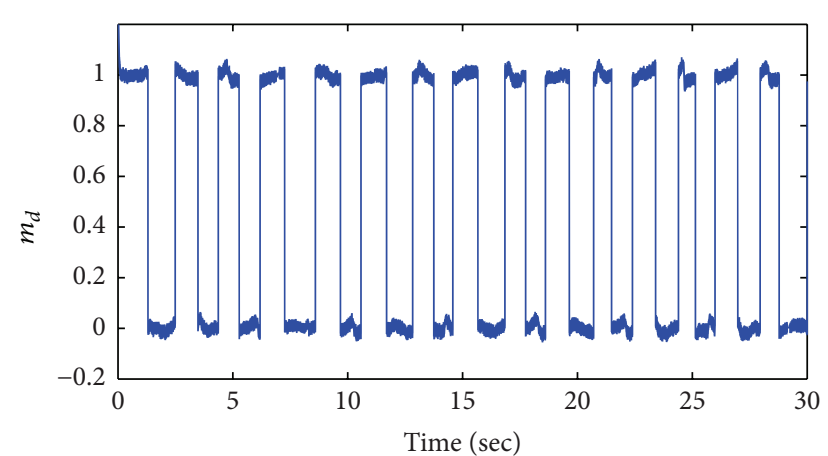

FIGURE 11: Simulation result of message decryption.

\section{Conflict of Interests}

The authors declare that there is no conflict of interests regarding the publication of this paper.

\section{Acknowledgment}

This research was supported by the International Research \& Development Program of the National Research Foundation of Korea (NRF) funded by the Ministry of Science, ICT and Future Planning of Korea (Grant no. K2012K1A3.A7A0305, FY 7508).

\section{References}

[1] G. Chen and X. Dong, From Chaos to Order: Methodologies, Perspectives and Applications, World Scientific, Singapore, 1998.

[2] T.-L. Liao and S.-H. Tsai, "Adaptive synchronization of chaotic systems and its application to secure communications," Chaos, Solitons and Fractals, vol. 11, no. 9, pp. 1387-1396, 2000.

[3] G. M. Mahmoud, E. E. Mahmoud, and A. A. Arafa, "On projective synchronization of hyperchaotic complex nonlinear systems based on passive theory for secure communications," Physica Scripta, vol. 87, no. 5, Article ID 055002, 2013.

[4] S.-H. Yu, C.-H. Hyun, W.-H. Kim, and M. Park, "Secure communication via active backstepping control and synchronization for new hyperchaotic systems," International Journal of Digital Content Technology and Its Applications, vol. 6, no. 10, pp. 276-286, 2012.

[5] C.-C. Cheng, Y.-S. Lin, and S.-W. Wu, "Design of adaptive sliding mode tracking controllers for chaotic synchronization and application to secure communications," Journal of the Franklin Institute, vol. 349, no. 8, pp. 2626-2649, 2012.

[6] V. H. P. Rodrigues and T. R. Oliveira, "Chaos synchronization applied to secure communication via sliding mode control and norm state observers," in Proceedings of the 13th International Workshop on Variable Structure Systems (VSS '14), pp. 1-6, IEEE, Nantes, France, July 2014.

[7] P. P. Singh, J. P. Singh, and B. K. Roy, "Synchronization and antisynchronization of Lu and Bhalekar-Gejji chaotic systems using nonlinear active control," Chaos, Solitons \& Fractals, vol. 69, pp. 31-39, 2014.

[8] A. N. Njah and U. E. Vincent, "Synchronization and antisynchronization of chaos in an extended Bonhöffer-van der Pol 
oscillator using active control," Journal of Sound and Vibration, vol. 319, no. 1-2, pp. 41-49, 2009.

[9] F. Wang and C. Liu, "Synchronization of unified chaotic system based on passive control," Physica D: Nonlinear Phenomena, vol. 225, no. 1, pp. 55-60, 2007.

[10] T. Sangpet and S. Kuntanapreeda, "Adaptive synchronization of hyperchaotic systems via passivity feedback control with timevarying gains," Journal of Sound and Vibration, vol. 329, no. 13, pp. 2490-2496, 2010.

[11] X. Tan, J. Zhang, and Y. Yang, "Synchronizing chaotic systems using backstepping design," Chaos, Solitons and Fractals, vol. 16, no. 1, pp. 37-45, 2003.

[12] B. A. Idowu, U. E. Vincent, and A. N. Njah, "Control and synchronization of chaos in nonlinear gyros via backstepping design," International Journal of Nonlinear Science, vol. 5, no. 1, pp. 11-19, 2008.

[13] J.-J. Yan, M.-L. Hung, and T.-L. Liao, "Adaptive sliding mode control for synchronization of chaotic gyros with fully unknown parameters," Journal of Sound and Vibration, vol. 298, no. 1-2, pp. 298-306, 2006.

[14] T.-H. S. Li, M.-Y. Hsiao, J.-Z. Lee, and S.-H. Tsai, "Interval type 2 fuzzy sliding-mode control of a unified chaotic system," Journal of Physics: Conference Series, vol. 96, no. 1, Article ID 012086, 2008.

[15] I. Yang and D. Lee, "Synchronization of chaotic gyros based on robust nonlinear dynamic inversion," Journal of Applied Mathematics, vol. 2013, Article ID 519796, 8 pages, 2013.

[16] I. Yang, D. Kim, and D. Lee, "A flight control strategy using robust dynamic inversion based on sliding mode control," in Proceedings of the AIAA Guidance, Navigation, and Control Conference, AIAA 2012-4527, Minneapolis, Minn, USA, August 2012.

[17] I. Yang, D. Lee, and D. S. Han, "Designing a robust nonlinear dynamic inversion controller for spacecraft formation flying," Mathematical Problems in Engineering, vol. 2014, Article ID 471352, 12 pages, 2014.

[18] H. P. Ren and W. C. Li, "Heteroclinic orbits in Chen circuit with time delay," Communications in Nonlinear Science and Numerical Simulation, vol. 15, no. 10, pp. 3058-3066, 2010.

[19] J. Georgie and J. Valasek, "Evaluation of longitudinal desired dynamics for dynamic-inversion controlled generic reentry vehicles," Journal of Guidance, Control, and Dynamics, vol. 26, no. 5, pp. 811-819, 2003. 


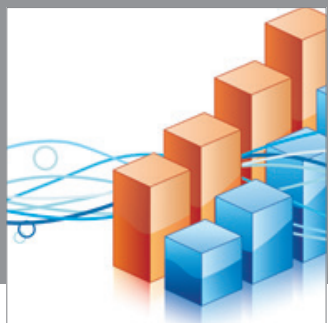

Advances in

Operations Research

mansans

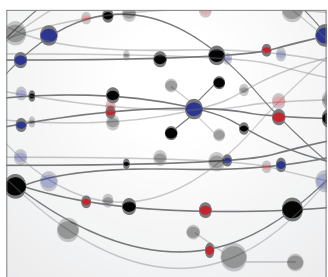

The Scientific World Journal
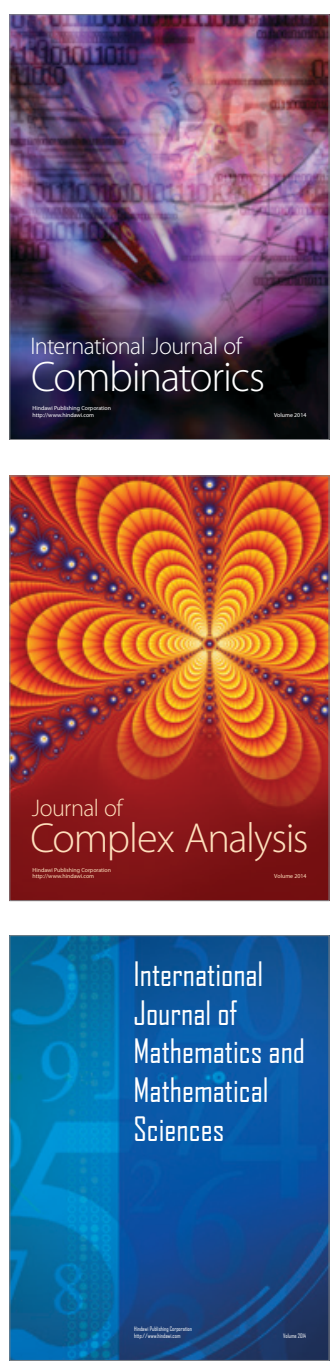
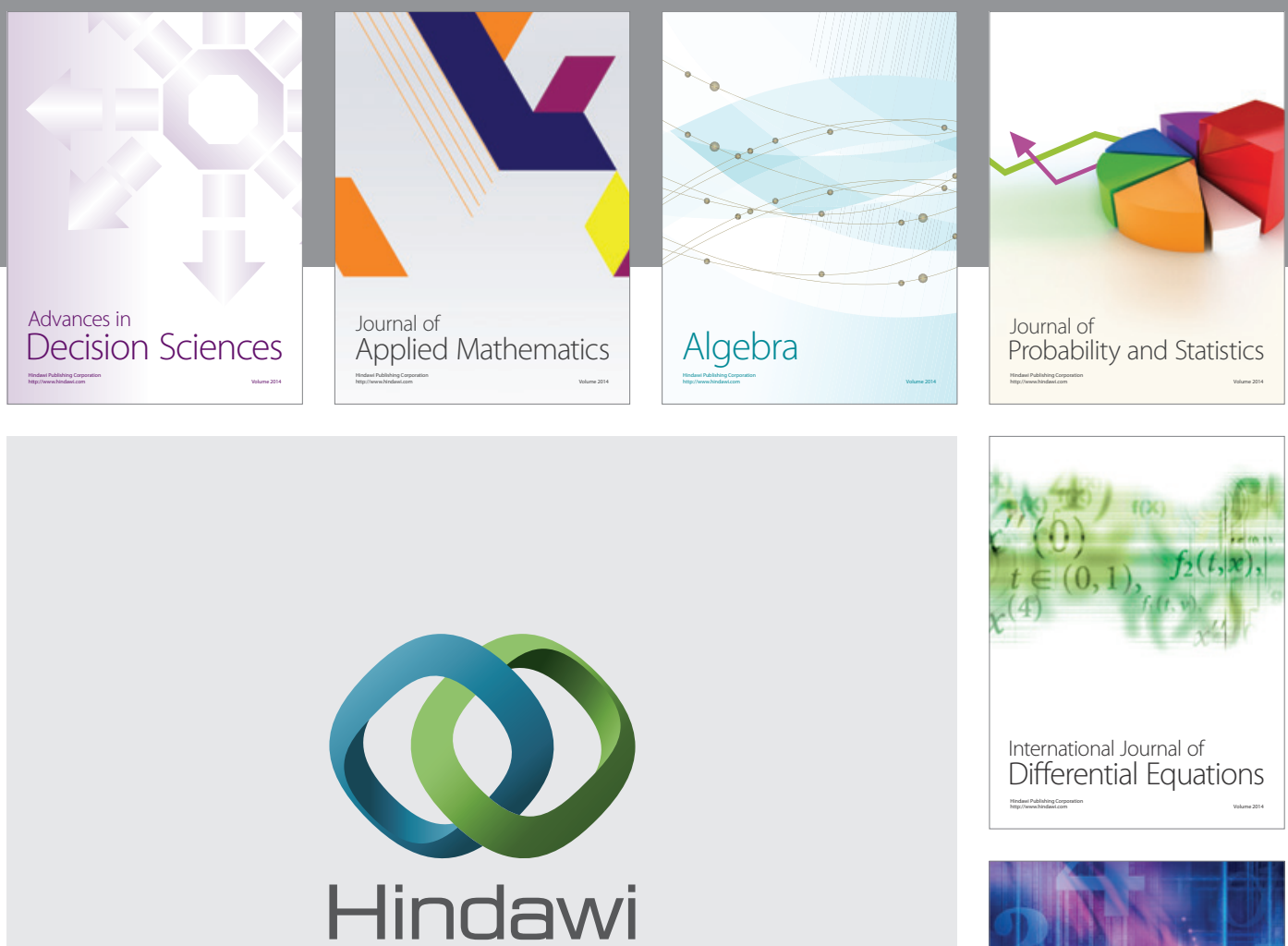

Submit your manuscripts at http://www.hindawi.com
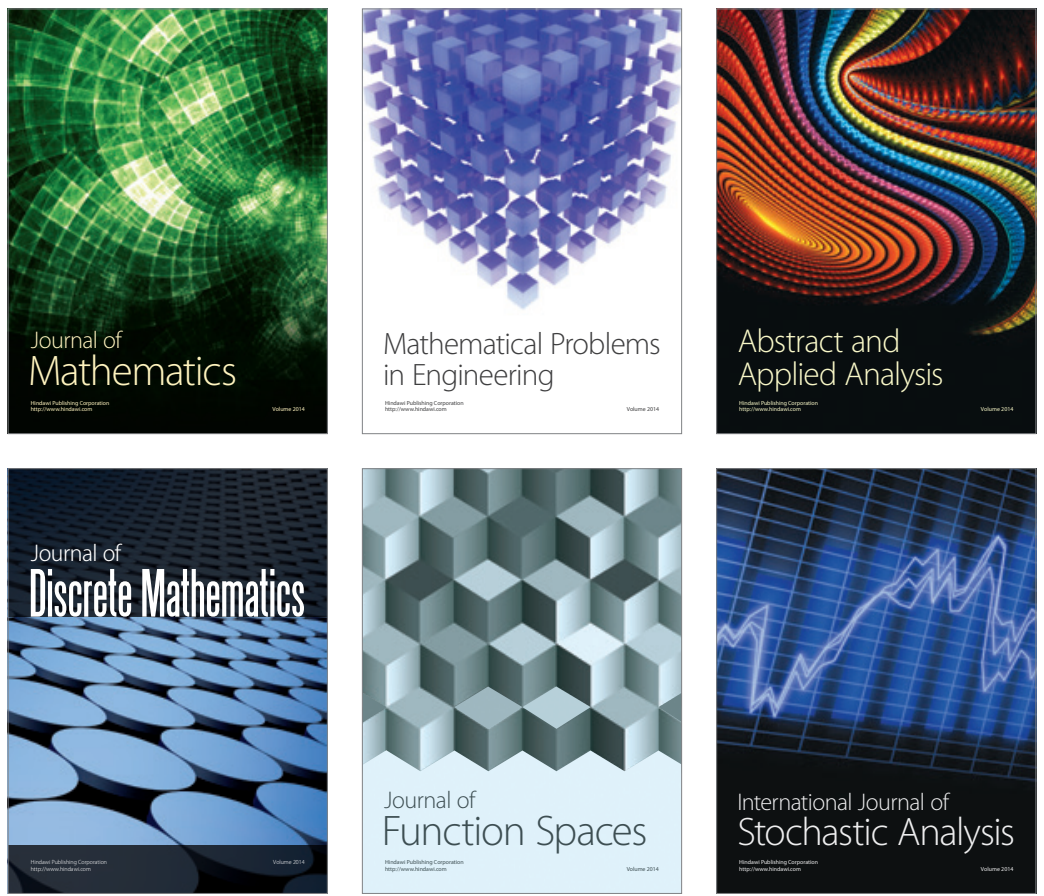

Journal of

Function Spaces

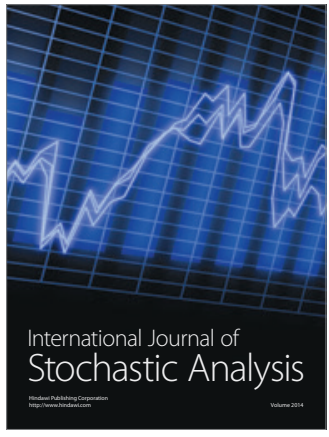

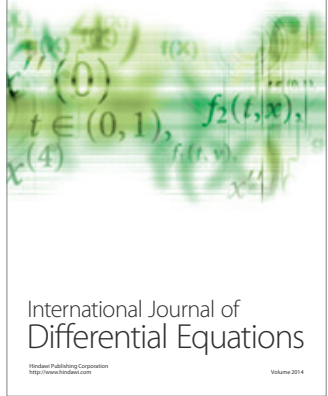
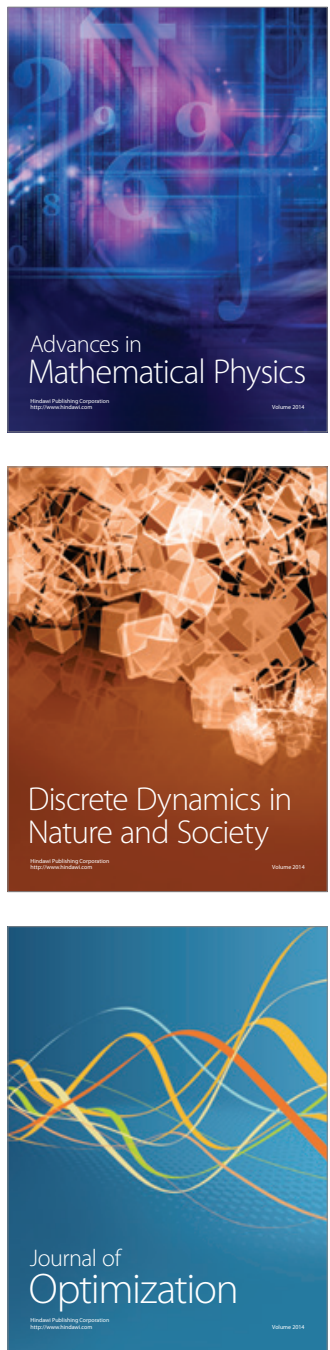